\title{
Variation of the Gravitational Constant in the Radiation-Dominated Universe
}

\author{
L. L. Williams \\ Konfluence Research, 6009 Olympic, Manitou Springs, CO 80829, USA \\ Correspondence should be addressed to L. L. Williams, lance@konfluence.org \\ Received 29 September 2011; Accepted 4 December 2011 \\ Academic Editor: Bijan Saha
}

Copyright () 2012 L. L. Williams. This is an open access article distributed under the Creative Commons Attribution License, which permits unrestricted use, distribution, and reproduction in any medium, provided the original work is properly cited.

The unification of classical electrodynamics and general relativity within the context of five-dimensional general relativity (Kaluza, 1921, and Thiry, 1948) contains a scalar field which may be identified with the gravitational constant, $G$. The field equations of this theory are solved under conditions of the Robertson-Walker metric for flat space, for a radiation-dominated universe-a model appropriate for the early history of our universe. This leads to a cosmology wherein $G$ is inversely proportional to the RobertsonWalker scale factor. This result is discussed in the context of the Dirac large number hypothesis and in the context of an expression for $G$ in terms of atomic constants.

\section{Introduction}

General relativity and classical electrodynamics are elegantly unified in the classical five-dimensional (5D) theory introduced by Kaluza [1], with the self-consistent field equations provided by Thiry [2] and Jordan and Müller [3]. It is important to distinguish the purely classical 5D theory from the quantum interpretations introduced by Klein [4]. In the purely classical theory, the fifth dimension is understood to be macroscopic, not compactified or microscopic. An early summary of the classical 5D theory was provided by [5].

The essence of the classical 5D theory is to posit a 15component 5D metric comprising the usual 10-component four-dimensional (4D) metric, the electromagnetic 4-vector potential, and a scalar field. Applying the 5D vacuum Einstein equations to the 5D metric yields the 4D Einstein equations with electromagnetic sources, plus the vacuum Maxwell equations. A fifteenth equation describes the scalar field. Applying the 5D geodesic hypothesis to the same metric yields the $4 \mathrm{D}$ geodesic equation with the Lorentz force term. To this framework is added the constraint, known as the cylinder condition, that none of the fields are observed to depend on the fifth coordinate.

It is unorthodox to seek fundamental unification in a theory which predates the quantum revolution. And it was the quantum discoveries which spurred abandonment of the purely classical 5D theory. But the perspective of 85 years has revealed to us what could not have been known when work on the classical theory was dropped: that a quantum theory of general relativity may be impossible, thereby blocking any attempt at a unification with quantum electrodynamics. If we are to unify general relativity with the other forces, we are obligated to look again at the common symmetries of general relativity and classical electrodynamics.

An intriguing result of 5D relativity is that the coupling of electromagnetic stress energy in the 4D Einstein equations is variable, depending on the scalar field. In other words, the gravitational "constant" will vary in a radiation-dominated universe. A radiation-dominated universe is an appropriate model of the early history of our own universe, from the Big Bang until the time of radiation-matter equality when the universe was around 50,000 years old [6]. In this paper the equations of $5 \mathrm{D}$ relativity are applied to a flat, radiationdominated universe. The modified Friedmann equation is derived, and the evolution of the Robertson-Walker scale factor and the gravitational constant are obtained. These results are contrasted with the standard model of the radiationdominated universe, and their implications are discussed.

\section{The Five-Dimensional Field Equations}

The fully self-consistent field equations of 5D relativity [2, 3 ] were written some 25 years after Kaluza [1] introduced 
the basic idea. Under the assumption that none of the field variables depend on the fifth coordinate, the field equations are given by

$$
\begin{gathered}
R_{\mu \nu}-\frac{1}{2} g_{\mu \nu} R=\frac{8 \pi G_{0}}{c^{4}} \phi^{2} T_{\mu \nu}^{\mathrm{EM}}-T_{\mu \nu}^{\phi}, \\
\nabla^{\mu} F_{\mu \nu}=-3 g^{\mu \alpha} F_{\mu \nu} \partial_{\alpha} \ln \phi, \\
g^{\alpha \beta} \nabla_{\alpha} \nabla_{\beta} \phi=\frac{4 \pi G_{0}}{c^{4}} \phi^{3} F_{\alpha \beta} F^{\alpha \beta},
\end{gathered}
$$

where $R_{\mu \nu}$ is the usual 4D Ricci tensor, $R$ is the Ricci scalar, $g_{\mu \nu}$ is the $4 \mathrm{D}$ metric, $G_{0}$ is the gravitational constant at a reference time, $\phi$ is the scalar field, $T_{\mu \nu}^{\mathrm{EM}}$ is the usual electromagnetic stress-energy tensor, $F_{\mu \nu}$ is the usual electromagnetic field tensor, $\nabla_{\alpha}$ is the covariant derivative, and greek indices span the 4 dimensions of space and time. The scalar field stress-energy tensor is defined by

$$
T_{\mu \nu}^{\phi} \equiv \phi^{-1}\left[\nabla_{\mu} \nabla_{\nu} \phi-g_{\mu \nu} g^{\alpha \beta} \nabla_{\alpha} \nabla_{\beta} \phi\right] .
$$

In the limit that the scalar field $\phi=1$, as originally assumed by Kaluza [1], (1) and (2) are the usual source-free Einstein and Maxwell equations.

The 5D unification leads to couplings between $g_{\mu \nu}$ and $F_{\mu \nu}$ through $\phi$. In particular, the factor in $\phi^{2}$ in the first term on the RHS of (1) will behave as a varying gravitational constant in terms of the coupling of spacetime to the electromagnetic stress energy. This, along with the stress-energy term in $\phi$, will modify the behavior of the Friedmann equation for a radiation-dominated universe.

\section{Radiation-Dominated Universe}

Now we apply these equations to the standard results for a radiation-dominated universe, for example, [7]. We solve (1) and (3) for the case of the Robertson-Walker metric describing a flat universe:

$$
c^{2} d \tau^{2}=c^{2} d t^{2}-a(t)^{2}\left(d r^{2}+r^{2} d \theta^{2}+r^{2} \sin ^{2} \theta d \psi^{2}\right),
$$

where $a(t)$ is the Robertson-Walker scale factor, presumed to depend only on the cosmic time coordinate $t$.

For (3) we invoke isotropy and homogeneity to assume that the scalar field, like $a$, depends only on $t$. The quantity on the RHS of (3), the electromagnetic Lagrangian, is proportional to the difference between the square of the electric field and the square of the magnetic field. For the early radiation-dominated universe, the electromagnetic field is expected to be a random photon field. In this case, the RHS of (3) should average to zero. Under these assumptions, (3) becomes

$$
g^{t t}\left(\ddot{\phi}+3 \frac{\dot{a}}{a} \dot{\phi}\right)=0,
$$

where the dots indicate single or double time derivatives. We can immediately write down the solution to (6) in terms of $a$ :

$$
\dot{\phi}=\frac{\kappa}{a^{3}}
$$

where $\kappa$ is a constant.
We turn now to the modified Friedmann equation, which is obtained from the $t t$ component of the Einstein equations, (1). The scalar field contribution from (4) is

$$
T_{t t}^{\phi}=-3 \frac{\dot{a}}{a} \frac{\dot{\phi}}{\phi} .
$$

The electromagnetic energy density will scale as $a^{-4}$ as usual [7]. Writing $T_{t t}^{\mathrm{EM}}=E_{0}\left(a_{0} / a\right)^{4}$, we obtain the Kaluzamodified Friedmann equation:

$$
\left(\frac{\dot{a}}{a}\right)^{2}=\frac{8 \pi G_{0}}{3 c^{4}} \phi^{2} E_{0}\left(\frac{a_{0}}{a}\right)^{4}-\frac{\dot{a}}{a} \frac{\dot{\phi}}{\phi} .
$$

Equations (6) and (9) now describe the radiationdominated universe in which gravity and electromagnetism are unified within $5 \mathrm{D}$ relativity. It may be verified that the following power-law solutions satisfy (6) and (9):

$$
\begin{gathered}
a=a_{0}\left(\frac{t}{t_{0}}\right)^{2 / 5}, \\
\phi=\phi_{0}\left(\frac{t}{t_{0}}\right)^{-1 / 5} .
\end{gathered}
$$

In these equations, $a_{0}$ and $\phi_{0}$ are constants which represent the values of $a$ and $\phi$, respectively, at the reference time $t_{0}$.

\section{Discussion}

When $\phi=1$, (9) reduces to the standard Friedmann equation for a flat, radiation-dominated universe. The scalar field equation (6) is a typical inflaton field equation without a potential term. The standard result for a radiationdominated universe has $a(t) \propto t^{1 / 2}$. So the effect in (10) of the scalar field is to flatten this dependence somewhat.

The reference time $t_{0}$ for this model is taken to be the time of matter-radiation equality, at the end of the radiationdominated era when the universe was approximately 50,000 years old [6]. Therefore, the Hubble parameter $\dot{a} / a$ evaluated at the reference time is not the value of the Hubble constant we have today. Likewise, the reference value $G_{0}$ of the gravitational constant is referred to $t_{0}$ and is not necessarily the value at the current epoch.

Note that the effective gravitational constant $G(t)$ in (9) is the quantity $G_{0} \phi^{2}$. Equations (10) and (11), therefore, imply

$$
G(t) \propto \frac{1}{a(t)}
$$

That is, the gravitational constant is inverse to the RobertsonWalker scale factor.

Of course, the variation of the gravitational constant, $\dot{G} / G$, has been extensively studied [8]. The variation in the current epoch has been constrained to a value of order $10^{-11}$ / year. Yet it remains for a later work to extend the predictions of this model to the current epoch.

Inferences about $\dot{G} / G$ in the early universe can be obtained from nucleosynthesis because the gravitational constant has some effect on the freeze-out temperature. There 
have been rather strict constraints of this nature placed on the variation of the gravitational constant [9]. These may indeed provide the evidence to falsify Kaluza's 5D relativity as a viable unification of gravity and electromagnetism. But such a conclusion may still be premature until the effects of matter and a cosmological constant are included in the model equation (9). Since nucleosynthesis occurs very early after the Big Bang, in the first few minutes, the model (6) and (9) should be interpreted as perhaps valid only between nucleosynthesis and radiation-matter equality.

The result (12) suggests an alternative formulation of the Dirac large number hypothesis. Let us now leave the specific case of the radiation-dominated universe to consider this alternative formulation. Recall that the original Dirac hypothesis [10] equated the ratio of the gravitational to electric force between a proton and electron to the ratio of the classical electron radius $r_{e}$ to the size of the visible universe:

$$
\frac{c}{r_{e}}\left(\frac{a}{\dot{a}}\right) \sim \frac{q^{2}}{G(t) m_{p} m_{e}} .
$$

Let us now modify the Dirac hypothesis by adjusting the LHS of (13) in accordance with (12):

$$
\frac{a(t)}{a_{0}} \sim \frac{q^{2}}{G(t) m_{p} m_{e}},
$$

where $a_{0}$ is here taken to be unity, with $a(t)>1$.

The theory does not provide us with the normalizing constant for (14) nor can we use the value Dirac used in (13). But to illustrate where we encounter the limits of the classical theory, let us consider the numeric expression for the gravitational constant discovered by Brandenburg [11]. Brandenburg proposes that the gravitational constant in the current epoch:

$$
G=\frac{\alpha q^{2}}{m_{p} m_{e}} \mathrm{e}^{-2\left(m_{p} / m_{e}\right)^{1 / 2}},
$$

where $\alpha$ is the fine structure constant, $m_{p}$ and $m_{e}$ are the proton and electron masses, and $q$ is the quantum of electric charge. This expression is accurate to within the measured uncertainty of the gravitational constant.

When Brandenburg's expression (15) is substituted into (14), we find

$$
a \sim \frac{1}{\alpha} \mathrm{e}^{2\left(m_{p} / m_{e}\right)^{1 / 2}} .
$$

Equation (16) is a remarkable parameterization of the current-epoch Robertson-Walker scale factor in terms of the electron-proton mass ratio and the fine structure constant. It has the mathematical form for the scale factor in a standard inflation model [7]: a large e-folding factor. The fine structure constant forms a seed length for the scale factor, and the e-folding depends only on the electron-proton mass ratio.

Of course, if Brandenburg's expression (15) is used in (13), the size of the visible universe also exhibits the exponential factor:

$$
c\left(\frac{a}{\dot{a}}\right) \sim \frac{\hbar}{m_{e} c} \mathrm{e}^{2\left(m_{p} / m_{e}\right)^{1 / 2}} .
$$

In this case, the Compton wavelength of the electron forms a seed-length for the e-folding. So the mere existence of the exponential factor is not enough to indicate whether $G \propto 1 / a$ or $G \propto \dot{a} / a$.

The point is that one may expect quantum effects to provide the normalization of this classical theory, as in (16); note that Planck's constant does not enter the e-folding expression. But the classical treatment can still provide a description of interesting dynamics, such as inflation or the variation of the gravitational constant. Furthermore, we may expect a deviation from the standard Dirac hypothesis of $G \propto \dot{a} / a$ while still preserving the basic idea of the cosmological variation of $G$.

\section{Conclusions}

The classical unification of electrodynamics and general relativity predicts that the electromagnetically induced deformation of spacetime is mediated by a scalar field. This scalar field will manifest as a varying gravitational constant, $G$, in terms of the coupling of the electromagnetic stress-energy to spacetime. When this system of equations is applied to the flat radiation-dominated universe with a Robertson-Walker metric, a model appropriate to our own early universe, one finds that $G$ varies inversely with the Robertson-Walker scale factor. This behavior suggests an alternative formulation of the Dirac large number hypothesis. A model of $G$ inversely proportional to the Robertson-Walker scale factor is consistent with an expression for $G$ in terms of other atomic constants. It is expected that quantum effects, and Planck's constant in particular, will set the fundamental length scales, but the classical theory can still predict interesting dynamics of cosmological parameters.

\section{References}

[1] T. Kaluza, Sitzungsberichte der K. Preussischen Akademie der Wissenschaften zu Berlin, vol. 966, 1921.

[2] M. Y. Thiry, "Geometry-The equations of Kaluza's unified theory," Comptes Rendus de l'Académie des Sciences, vol. 226, p. 216, 1948.

[3] P. Jordan and C. Müller, "Uber die feldgleichungen der gravitation bei variabler "Gravitationkonstante", Zeitschrift Fur Naturforschung, vol. 2a, pp. 1-2, 1947.

[4] O. Klein, "The atomicity of electricity as a quantum theory law," Nature, vol. 118, no. 2971, p. 516, 1926.

[5] V. Bargmann, "Relativity," Reviews of Modern Physics, vol. 29, no. 2, pp. 161-174, 1957.

[6] J. A. Frieman, M. S. Turner, and D. Huterer, "Dark energy and the accelerating universe," Annual Review of Astronomy and Astrophysics, vol. 46, pp. 385-432, 2008.

[7] J. A. Peacock, Cosmological Physics, Cambridge University Press, New York, NY, USA, 1999.

[8] G. T. Gillies, “The Newtonian gravitational constant: recent measurements and related studies," Reports on Progress in Physics, vol. 60, no. 2, pp. 151-225, 1997.

[9] J.-P. Uzan, "The fundamental constants and their variation: observational and theoretical status," Reviews of Modern Physics, vol. 75, no. 2, pp. 403-455, 2003. 
[10] P. A. M. Dirac, "The cosmological constants," Nature, vol. 139, no. 3512, p. 323, 1937.

[11] J. E. Brandenburg, "Unification of gravity and electromagnetism in the plasma universe," IEEE Transactions on Plasma Science, vol. 20, p. 944, 1992. 

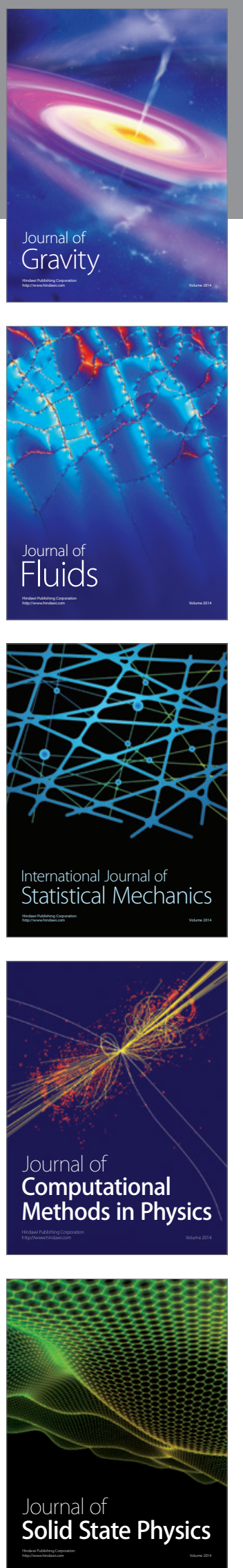

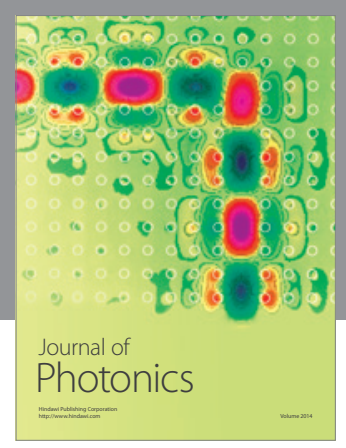

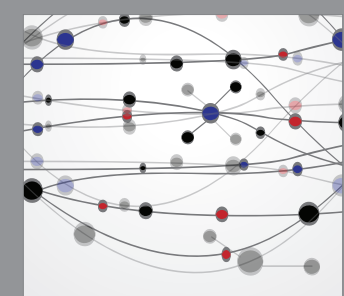

The Scientific World Journal
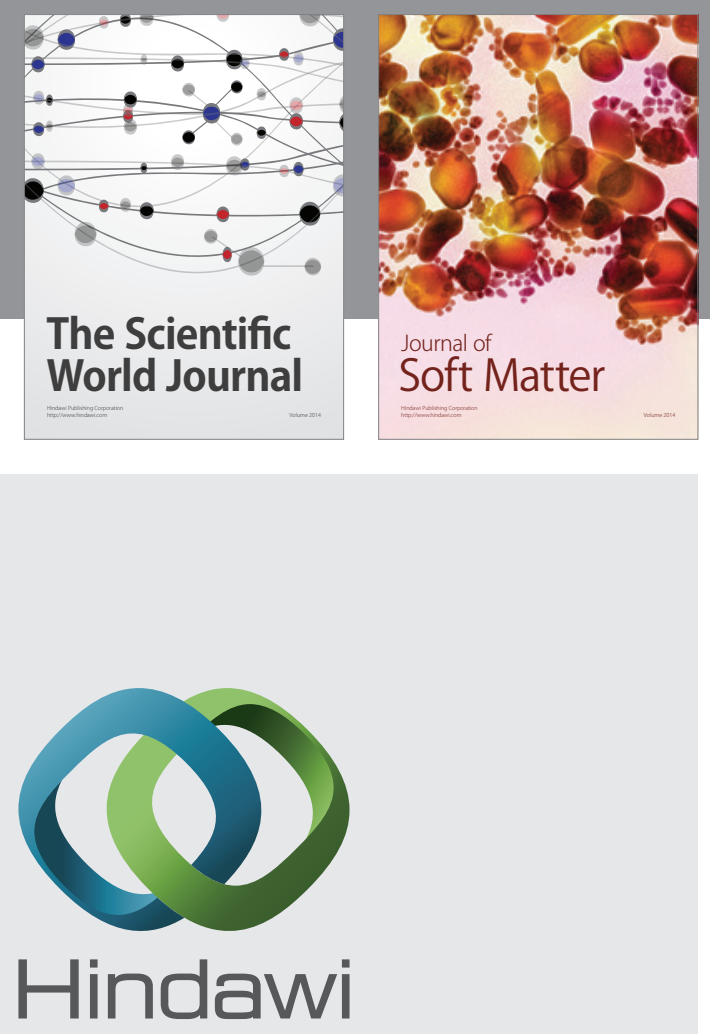

Submit your manuscripts at

http://www.hindawi.com
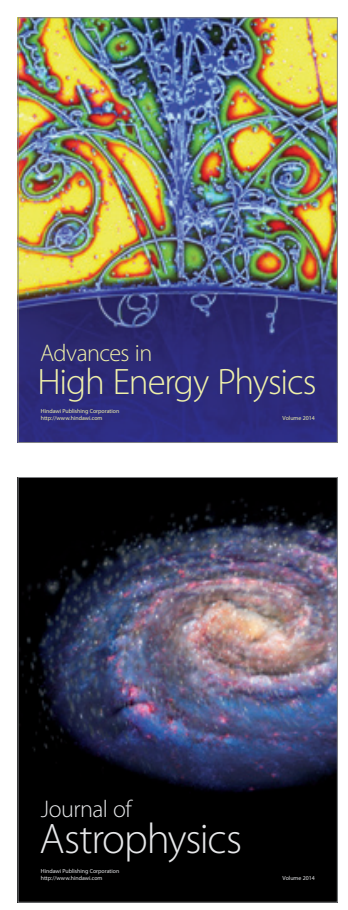
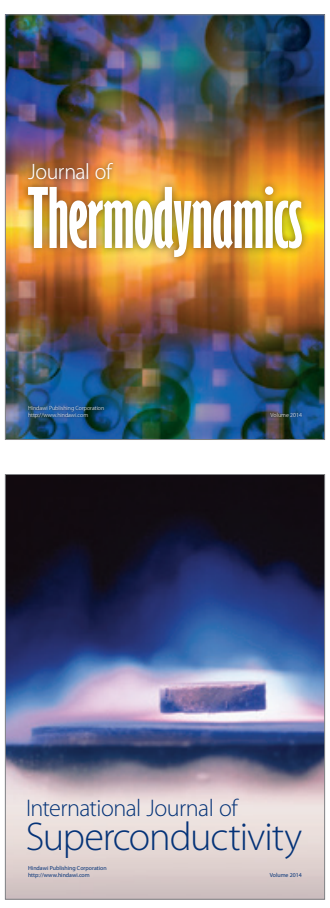
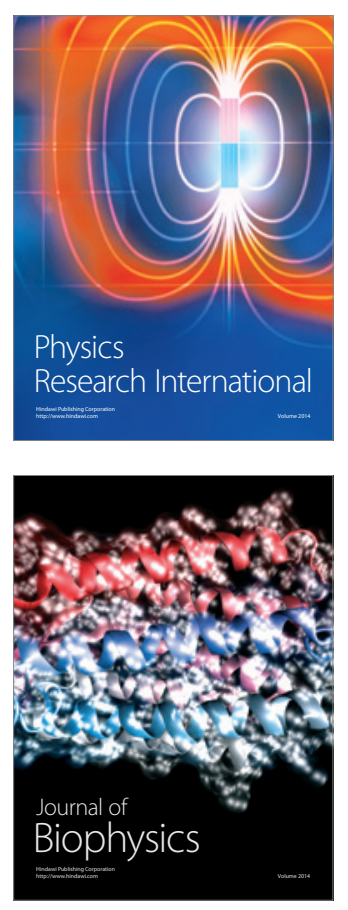
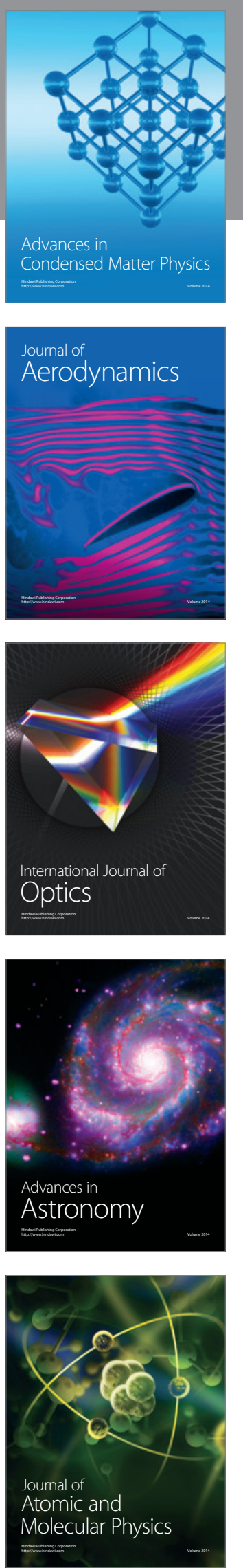